PharmacoEconomics \& Outcomes News 887, p3 - 18 Sep 2021

\title{
Acute respiratory distress syndrome costly but studies vary in estimates and quality
}

A systematic review of 22 economic studies has found that acute respiratory distress syndrome (ARDS) is a costly condition, but with wide variation in cost estimates and quality of studies, say researchers from Canada.

Two researchers independently conducted a review of MEDLINE, Embase, Central and EconLit databases for articles that reporting cost data from an original publication in adult patients with ARDS. They then extracted data relating to costs, methodology, healthcare system type, economic perspective, and clinical data. Publication quality was assessed using a modified version of the Quality of Health Economic Studies Instrument.

Of the 22 studies, 13 were from the USA, three from the UK, three from Canada, and one each from Taiwan, Brazil and Finland. Ten examined inpatient costs only, 7 also included post-discharge costs, and two examined lost productivity costs. The studies included a wide range of healthcare systems, economic perspectives, costing methodology and timeframes. Mean inpatient cost values were highest in lower-quality publications, in US health systems and in trauma cohorts, ranging from $\$ \cup S 8476$ to $\$ 547974^{*}$. Outpatient costs were highest in publications with higher readmission rates, longer follow-up duration and in US health systems. Total mean costs at 1 year ranged from \$53 861 in one UK study, to \$134 579 in a US study. In the studies taking a societal perspective (one from the USA and one from the UK), there was much higher overall third party healthcare costs relative to the societal costs; however, there were methodological flaws in these studies, note the authors.

"There is a need for comprehensive costing data in this population to inform healthcare systems especially in light of the current pandemic related to COVID-19" they conclude.

* Costs were converted to 2021 values.

Boucher PE, et al. The cost of the Acute Respiratory Distress Syndrome: A systematic review. Chest : 31 Aug 2021. Available from: URL: http://doi.org/10.1016/ j.chest.2021.08.057 